Article

\title{
Friction Reduction in Unidirectional Lubricated Sliding Due to Disc Surface Texturing
}

\author{
Slawomir Wos@, Waldemar Koszela, Andrzej Dzierwa and Pawel Pawlus * \\ Faculty of Mechanical Engineering and Aeronautics, Rzeszow University of Technology, Powstancow Warszawy \\ 8 Street, 35-959 Rzeszow, Poland; wosslawomir@prz.edu.pl (S.W.); wkktmiop@prz.edu.pl (W.K.); \\ adzierwa@prz.edu.pl (A.D.) \\ * Correspondence: ppawlus@prz.edu.pl; Tel.: +48-17865-1183
}

Received: 26 January 2020; Accepted: 27 February 2020; Published: 29 February 2020

check for updates

\begin{abstract}
Surface texturing is an option of surface engineering resulting in reduction of friction due to the creation of isolated dimples or grooves on sliding surfaces. The purpose of this work is to find the effect of the groove bottom profiles on the reduction of the friction force. Investigations were conducted using an Optimol SRV5 tribotester equipped with a pin-on-disc module. A disc made of $42 \mathrm{CrMo} 4$ steel, with a $100 \mathrm{~mm}$ diameter acted as a sample. A counter-sample was made of the same material, however, its diameter was $20 \mathrm{~mm}$. The sliding conditions were selected to be similar to those of a sliding crankpin bearing pad operating in a high-performance internal combustion engine. Surface texturing was found to be beneficial for a reduction in the friction coefficient up to $55 \%$ in comparison to the untextured disc. Tribological performances of discs with spiral groove patterns were better than those with a radial layout. In most cases the convergent profile of the groove bottom was superior to the dam shape.
\end{abstract}

Keywords: surface texturing; pin-on-disc; conformal contact; friction force; lubricated sliding

\section{Introduction}

Surface texturing is one of the fundamental methods of changing sliding conditions under lubrication. As a result of surface texturing a decrease in wear and better efficiency of mechanisms can be achieved [1-5]. Typically, beneficial effects of surface texturing in mixed and boundary lubrications are larger than those found under full film lubrication [4]. Many years ago a surface directionality was found to be a way to improve tribological performances of sliding elements [6]. Therefore, structures with parallel or cross-hatched grooves [7-10] were created. Textured surfaces with isolated dimples are also used [11-15].

The orientation of grooves to the sliding direction is very important from the tribological point of view. Petterson and Jacobson [16] found that in a regime of starved lubrication grooves positioned perpendicularly to the sliding direction led to smaller frictional resistance compared to their location parallel to the direction of motion. Perhaps when the ball slid along the grooves there was a small probability for a very small area of contact to cross an oil reservoir. Moronuki and Furukawa [17] obtained similar results after sliding tests between textured $\mathrm{Si}$ and planar $\mathrm{Si}$ in a low contact pressure regime. These results were caused by minimizing the adhesion force by the grooves perpendicular to sliding due to water capillary. The experimental research conducted by Yuan et al. [18] revealed that under a low contact pressure the grooves orthogonal to the movement direction caused more decrease in the coefficient of friction that those oriented along the sliding direction. Probably the strongest hydrodynamic lift was offered when the grooves were perpendicular to the sliding direction. Ren et al. [19] obtained the same reason of the results of tribological investigations. They achieved the beneficial effect of perpendicular orientation of grooves to the sliding direction on the frictional resistance 
under a slide-to-roll contact [19]. According to Zum Gahr et al. [20] the grooves perpendicularly aligned to the direction of motion led to smaller friction force; this behavior is related to higher oil film thickness than obtained for grooves parallel to the sliding direction. According to Costa and Hutchings [21] and Duarte et al. [22] parallel textures guide the lubricant away from the contact leading to a smaller film thickness and, thus, higher coefficient of friction. The authors of papers $[10,23,24]$ found that a decrease in the honing angle led to lower coefficient of friction of cylinder liner-piston ring pack. Probably a small honing angle enhanced hydrodynamic action.

Generally, for one-directional surfaces, the main direction should be oriented orthogonally to the direction of motion.

The effect of oil pockets orientation to the sliding direction for the chevron-shaped pattern was studied by the authors of papers: [25-28]. They found that chevron pattern pointing along the direction of motion could lead to film thickness growth. Wang [29] and Galda et al. [30] observed that orientation of triangle-shaped oil pockets and dimples of long drop shape, respectively, had a substantial effect on tribological behaviors of sliding pairs. The lowest friction force was achieved when one side border orthogonal to the sliding direction first entered the contact zone.

The optimization of the dimple bottom profiles was done experimentally. Lu et al. tested triangular [31] and squared [32] sloped dimples in lubricated line contact. The bottoms with the converging shapes helped to minimize friction by generating hydrodynamic lift. Galda et al. [30] obtained the same finding after testing dimples of the long drop shape with the sloped bottoms.

Various fabrication techniques were used for surface texturing [33]. Laser texturing is the most widely used, because this method seems to be the most flexible and efficient $[1,11,13-15,34-36]$. Abrasive jet machining can be an alternative to laser texturing [28,37-39]. The other methods include electrochemical etching, lithography [12], photochemical texturing, burnishing (embossing) [30,40] and some kinds of machining (honing [8-10] or grinding). A sharp tip can be also used for creating grooves [41]. This process can be facilitated using machines equipped with CNC.

The main aim of this work is to find the effect of the groove bottom profiles on the friction reduction, based on the diverging and converging characteristics of the textures. Similar experimental investigations were done rarely.

Surface texturing highly depends on the application; it should be dedicated to a special tribological system. The sliding conditions used in this work correspond to those of sliding crankpin bearing pad operating in a high-performance internal combustion engine. Such pads are typically threatened by severe wear, due to starved lubrication and high temperature in a race motorcycle engine. The attempt was done to achieve low friction, wear and heat generation due to surface texturing.

\section{Materials and Methods}

A disc made of $42 \mathrm{CrMo} 4$ steel with a $100 \mathrm{~mm}$ diameter acted as a sample. A counter-sample was made of the same material, however, its diameter was $20 \mathrm{~mm}$. Samples and counter-samples were heat treated to receive hardness of $40 \mathrm{HRC}$ for the sample and $35 \mathrm{HRC}$ for the counter-sample. Textured discs had higher hardness than the counter-samples to increase their wear resistances. Due to it wear of the textured sample was lower than that of the counter-sample leading to smaller friction and prolonged life of the tribological system. The experiments were carried out using an Optimol SRV5 tribotester (produced by: Optimol Instruments Prüftechnik GmbH, Flößergasse 3, D-81369 München, Germany) equipped with a rotary module. The method of fixing the counter specimen was modified to ensure better aligning of the cooperating surfaces and eliminate fixing errors (Figure 1). The normal load was $50 \mathrm{~N}$, the rotational speed was $500 \mathrm{rpm}$ and the friction radius was $20 \mathrm{~mm}$. The attempt was done to ensure the closest sliding parameters to those existed inside the motorcycle engine during start phase. Tests were carried out in temperature of $30^{\circ} \mathrm{C}$. Before each test the sample and the holder were heated up and hold in $30^{\circ} \mathrm{C}$ for $5 \mathrm{~min}$ to stabilize the temperature. Temperature sensor was located under the rotating disc inside the holder. Therefore, the heat generated by friction force was 
impossible to monitor precisely due to the heat dissipation. Surface textures of contacting elements were measured by a white light interferometer Talysurf CCI Lite.

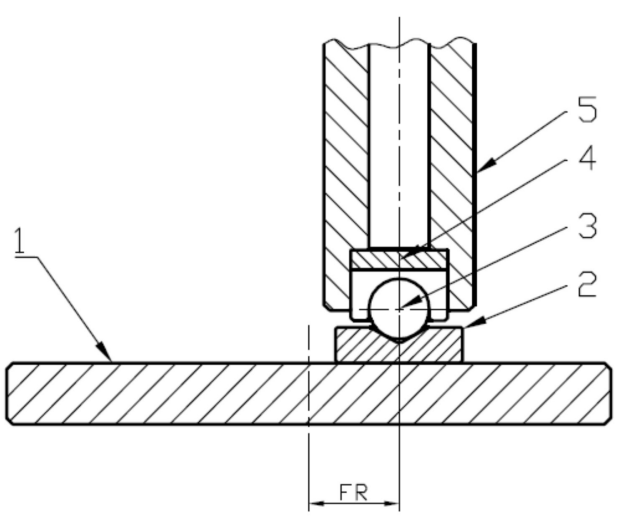

(a)

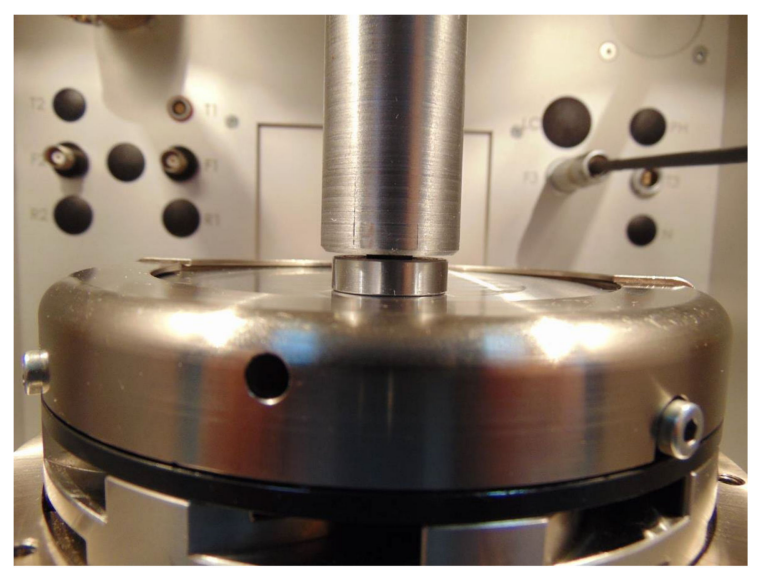

(b)

Figure 1. Scheme of counter-sample mounting; 1-sample (rotating disc), 2-self-aligning counter-sample, 3-ball bearing, 4-ball bearing holder, 5-load transmitting rod, FR-friction radius (a); view of test chamber (b).

In the beginning of each test a volume of $5 \mathrm{~mL}$ of semisynthetic motorcycle oil 10W40 T4 was supplied to the test zone. There are the following parameters of a lubricant: kinematic viscosity $66 \mathrm{~mm}^{2} / \mathrm{s}(66 \mathrm{cSt})$ at $40{ }^{\circ} \mathrm{C}$, and $13.5 \mathrm{~mm}^{2} / \mathrm{s}(13.5 \mathrm{cSt})$ at $100{ }^{\circ} \mathrm{C}$, ignition temperature $180{ }^{\circ} \mathrm{C}$, and a freezing point of $-18{ }^{\circ} \mathrm{C}$. This specific oil was selected due to its designation to high-performance motorcycle engines. Base surfaces of samples were ground and some of them were tested without texturing as reference samples, their roughness parameter Ra was $0.25 \pm 0.01 \mu \mathrm{m}$. Surface texturing was made by precise milling. The milling process was chosen due to the great accessibility of CNC mills. We tried to present the possibilities of using standard CNC machines in surface texturing instead of using other techniques, like laser surface texturing, electrical discharge machining or etching, in which additional devices are required. Moreover, thanks to careful production process planning it is possible to create grooves by adding only one additional operation to CNC machine. That approach does not lead to a high increase in the production cost. Three types of the milling process was used. The first one required 5 axis CNC milling machine, where mounted disc was tilted by $0.5^{\circ}$ from the horizontal direction. This approach helped to form a groove of with a sharp edge on one side and a convergent bottom on the other side (Figure 2).
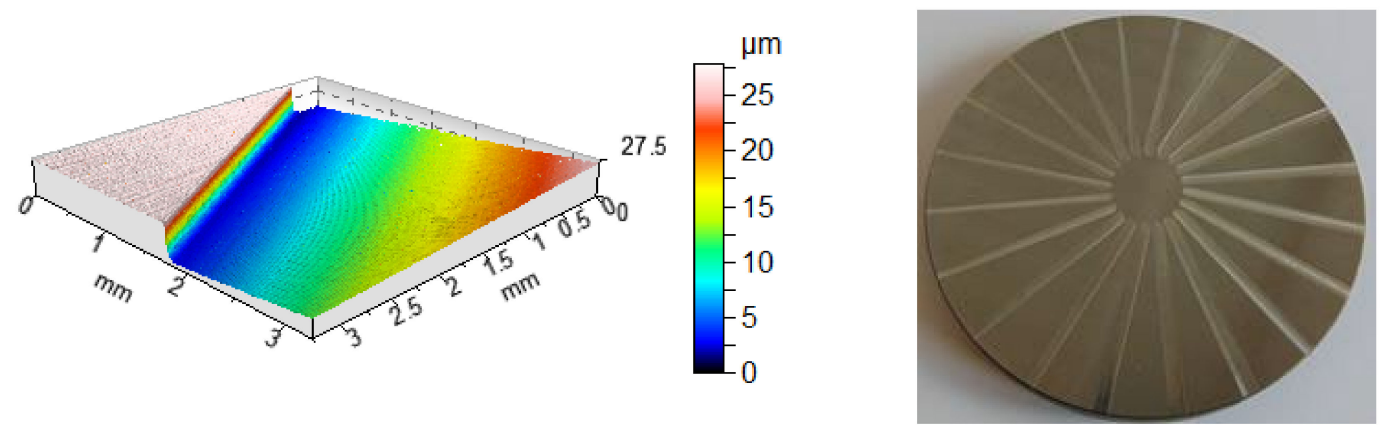

Figure 2. Isometric view of the bottom of the groove of a sharp edge on one side and a convergent bottom (left) and sample view with straight radial grooves of type 1 (right).

Other two types of created grooves were made with the use of sharp milling tool of 3 mm diameter and ball milling tool of $6 \mathrm{~mm}$ diameter. With application of sharp milling tool positioned vertically to a 
sample the both sides sharp edge groove was obtained. This type acted in lubricated sliding conditions as the both side dam (divergent) (Figure 3a).

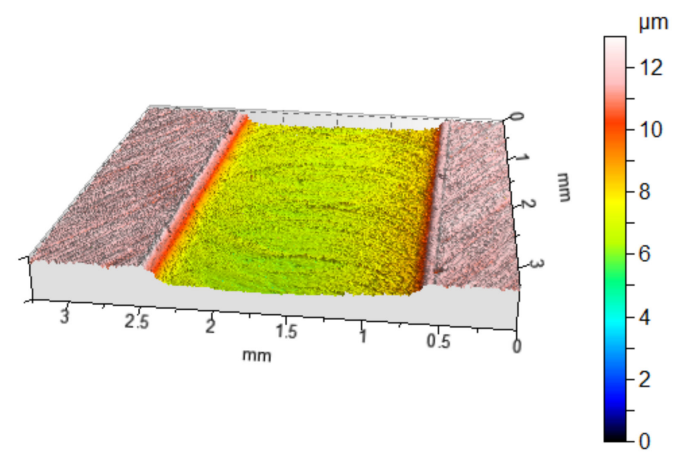

(a)

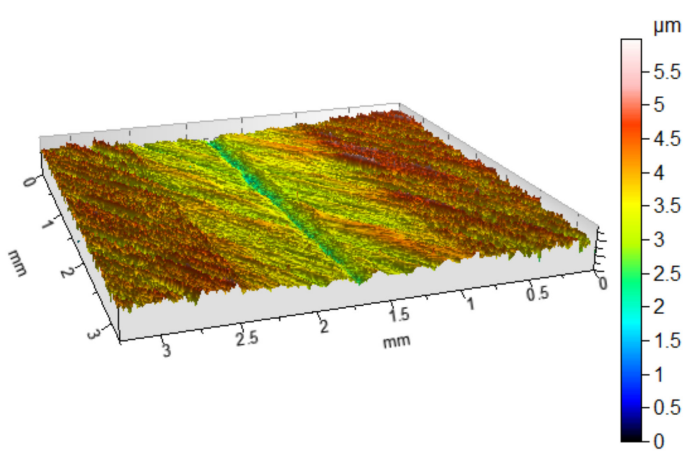

(b)

Figure 3. Isometric view of a surface created: (a) using a sharp mill cutter with sharp edges from both sides and (b) made with the use of a ball mill cutter.

Other type of a groove was made using a ball mill cutter; due to spherical shape of the tool light slopes at the edges of the groove were obtained, its depth slowly increased from the sides to achieve maximum value at the groove center. This geometry was called both side convergent (Figure 3b).

Figure 4 shows schemes of bottom profiles of grooves with sliding direction of the counter-sample.

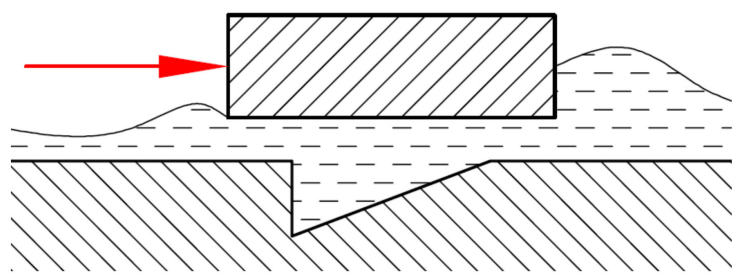

(a)

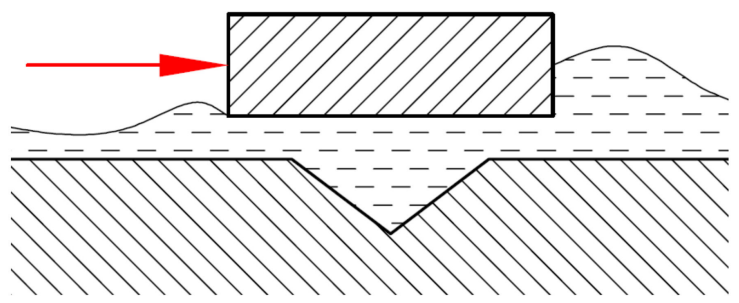

(c)

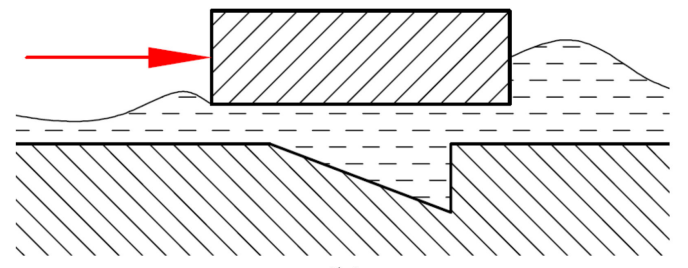

(b)

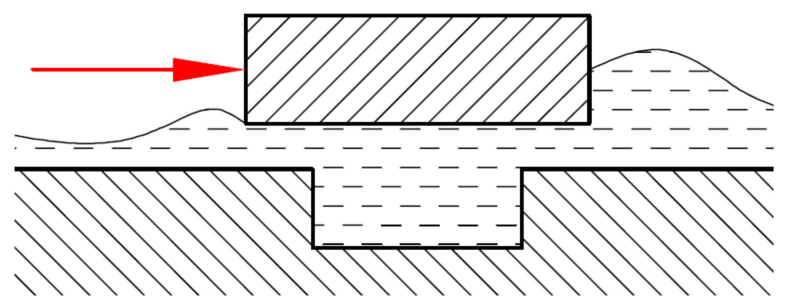

(d)

Figure 4. Schemes of bottom profiles of grooves with marked sliding direction of the counter-sample.

(a) Convergent; (b) Dam; (c) Both side convergent; (d) Both side dam.

Discs with radial pattern of the grooves were called discs of type 1 (Figure 2). The spiral layout was created to gather the lubricant to the contact zone and then to minimize oil loss. Discs of types 2 and 3 originated from different parts of Archimedes curve; the starting point of the curve was located at a center of the disc. Discs of type 4 and 5 were made from the same parts of the curve, however, starting points of the grooves were spaced from the centers of discs by $10 \mathrm{~mm}$.

Due to milling tool positioning during five-axis machining a problem with precision occurred. Shaping a spiral groove with an asymmetric profile was possible, however, in this case the groove had different depths at its whole length. This problem was caused by adding positioning accuracies of all five axes during simultaneous movement. This was the reason why spiral grooves had only two bottom shapes: both side convergent and both side dam. These shapes required simultaneous movement in only two axes, in the third axis the depth of material cut was set before spiral shape 
milling. The bottom profiles of the textures were defined by the shape of used mills. The convergent bottom shapes were created to form lubrication wedges. The dam shapes are typically formed by laser surface texturing or etching, therefore they were also taken into consideration.

Figure 5 shows photos of discs with spiral patterns of grooves and dimensions of guiding lines of these grooves from the discs of types $2-4$. There were 20 grooves on each disc. The number of test repetitions was at least 3.

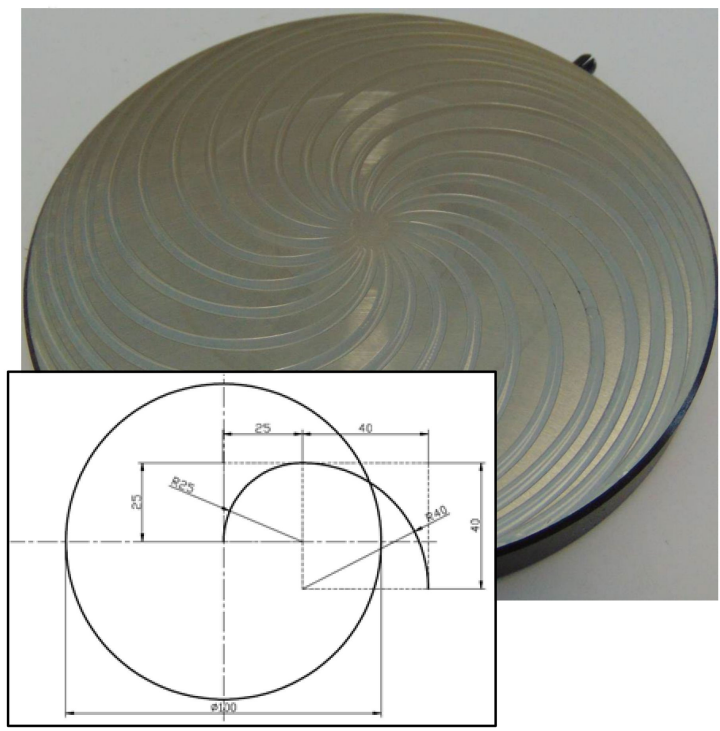

(a)

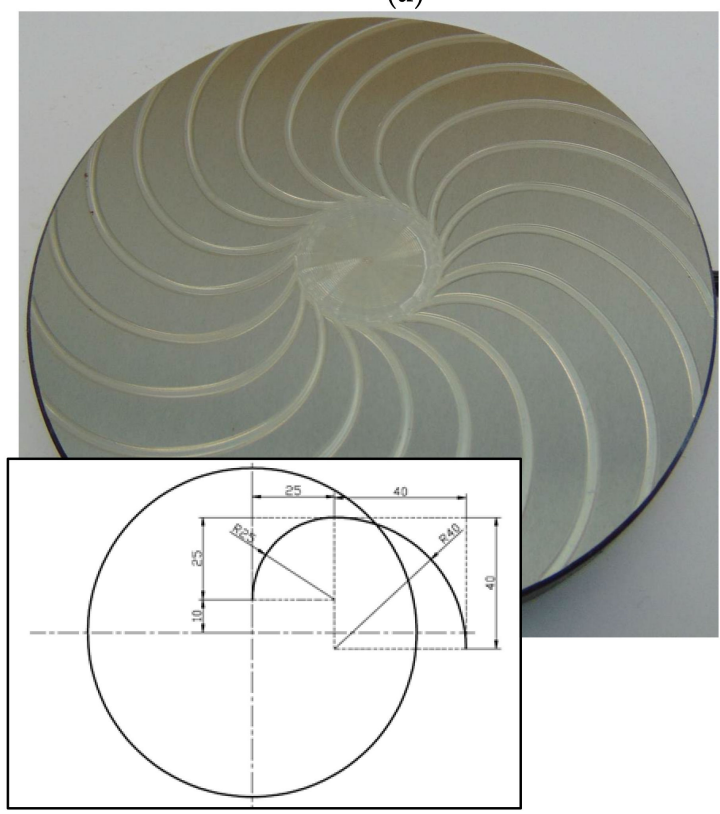

(c)

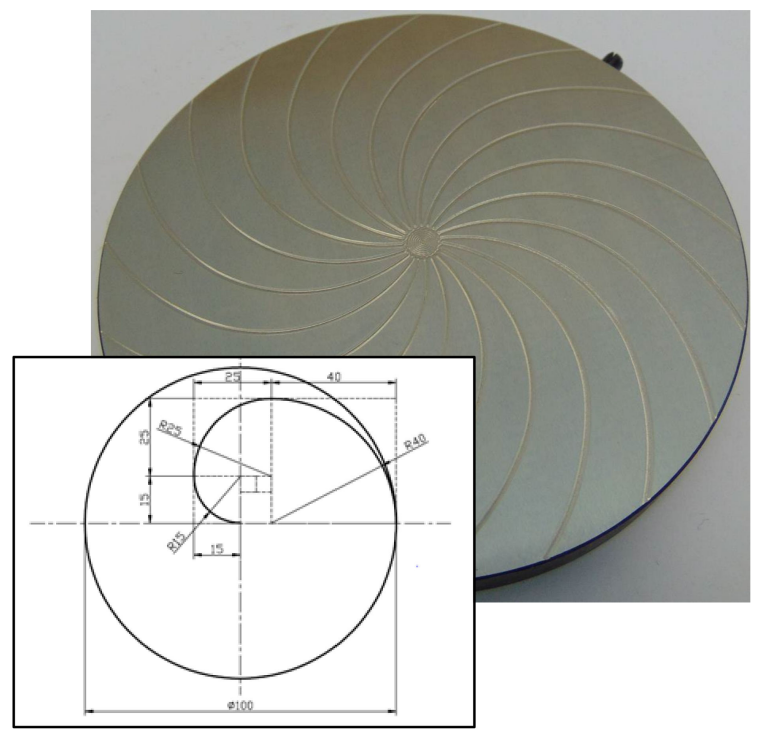

(b)

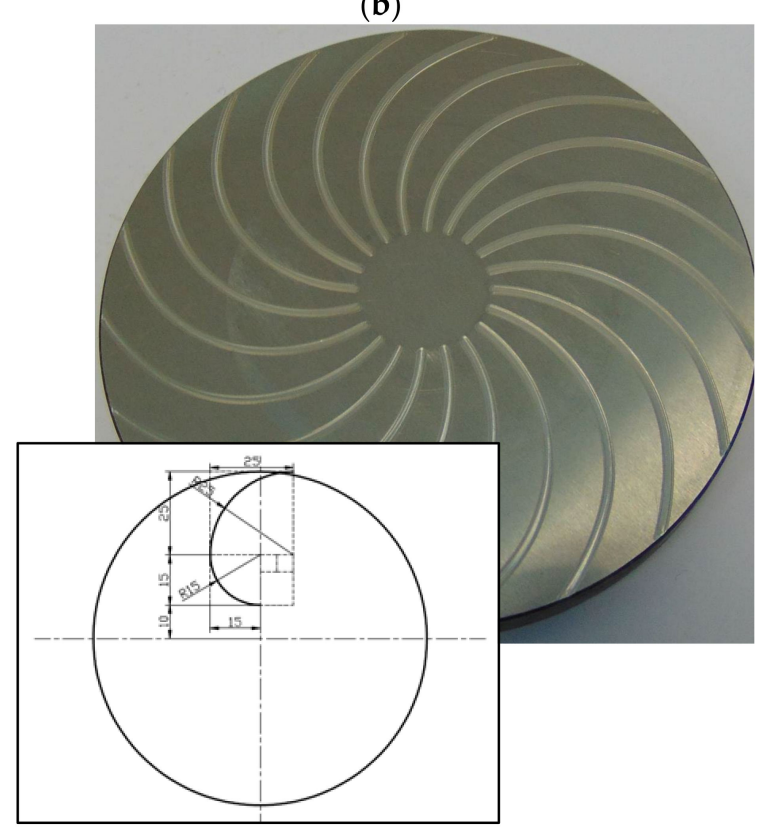

(d)

Figure 5. Views of discs of types 2 (a), 3 (b), 4 (c), and 5 (d) with dimensions of guiding lines.

Figure 6 shows isometric views of selected grooves from spiral discs. Table 1 presents dimensions of grooves from various textured discs. 

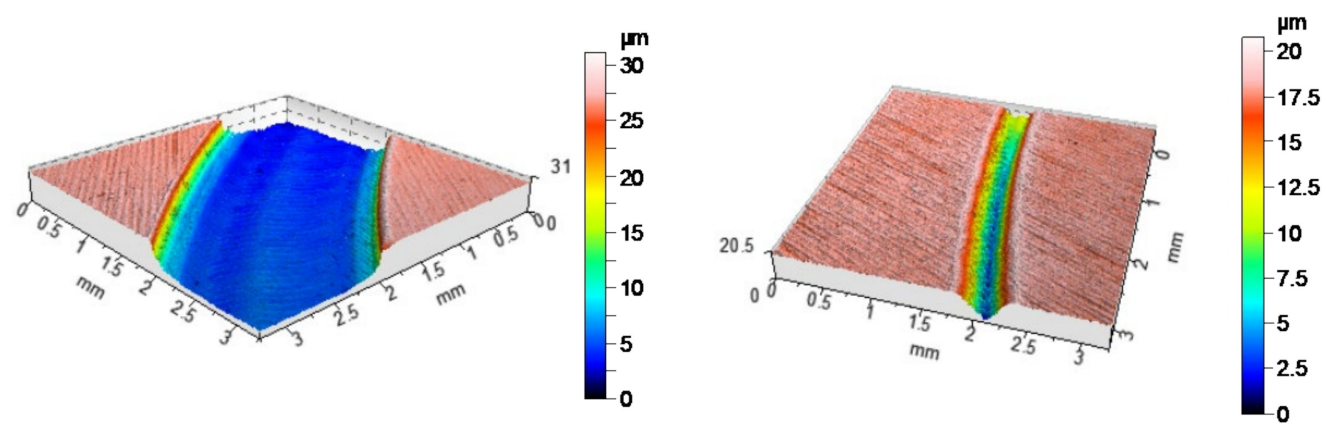

Figure 6. Isometric views of grooves from discs of type 2: both side dam (left) and both side convergent (right).

Table 1. Sizes of grooves from various textured discs.

\begin{tabular}{|c|c|c|c|}
\hline Kind of the Groove & $\begin{array}{l}\text { Width of the Groove } \\
{[\mathrm{mm}]}\end{array}$ & $\begin{array}{l}\text { Depth of the Groove } \\
{[\mu \mathrm{m}]}\end{array}$ & $\begin{array}{l}\text { Average Area of the Hole in the } \\
\text { Cross Section }\left[\mathrm{mm}^{2}\right]\end{array}$ \\
\hline \multicolumn{4}{|c|}{ Type 1} \\
\hline Convergent & $3.1 \pm 0.1$ & $24.7 \pm 1.2$ & 0.040900 \\
\hline Dam & $3.15 \pm 0.05$ & $25.4 \pm 1.4$ & 0.041600 \\
\hline Both side convergent & $1.6 \pm 0.1$ & $4.5 \pm 0.4$ & 0.001012 \\
\hline Both side dam & $1.95 \pm 0.05$ & $28.8 \pm 1.8$ & 0.042000 \\
\hline \multicolumn{4}{|c|}{ Type 2} \\
\hline Both side convergent & $0.61 \pm 0.1$ & $17.5 \pm 3$ & 0.003747 \\
\hline Both side dam & $1.9 \pm 0.05$ & $23 \pm 3$ & 0.039697 \\
\hline \multicolumn{4}{|c|}{ Type 3} \\
\hline Both side convergent & $0.6 \pm 0.1$ & $25.3 \pm 1.8$ & 0.003677 \\
\hline Both side dam & $2.1 \pm 0.05$ & $30.4 \pm 2.4$ & 0.044300 \\
\hline \multicolumn{4}{|c|}{ Type 4} \\
\hline Both side convergent & $0.6 \pm 0.1$ & $25.7 \pm 1$ & 0.003201 \\
\hline Both side dam & $1.9 \pm 0.05$ & $30 \pm 1$ & 0.037354 \\
\hline \multicolumn{4}{|c|}{ Type 5} \\
\hline Both side convergent & $0.56 \pm 0.1$ & $26.3 \pm 2.2$ & 0.002331 \\
\hline Both side dam & $1.95 \pm 0.05$ & $16.9 \pm 1.6$ & 0.031540 \\
\hline
\end{tabular}

One can see from the analysis of Table 1 that depths of the grooves were typically between 17 and $34 \mu \mathrm{m}$; both side convergent grooves from disc of type 1 of the lowest depth $(4.5 \mu \mathrm{m})$ were the exceptions. For discs with spiral patterns of grooves the main difference between grooves called both side convergent and both side dam was width which was much larger in the latter case. The changes of width affected also the area of the hole in the cross-section. Due to a difference in depths this area of both side dam groove of disc 1 was also larger than that of both side convergent groove.

\section{Results and Discussion}

Figure 7 shows curves presenting friction forces versus time for the sliding pair containing the untextured disc. Due to instability of the friction force, the number of test repetitions was 4 . One can see that the friction force after initial fluctuations increased as test progressed and obtained a stable value after about 170 seconds. The final value of the friction force was between 3 and $6 \mathrm{~N}$. In the middle and final parts of tests large variations of the friction forces also occurred in most cases. 


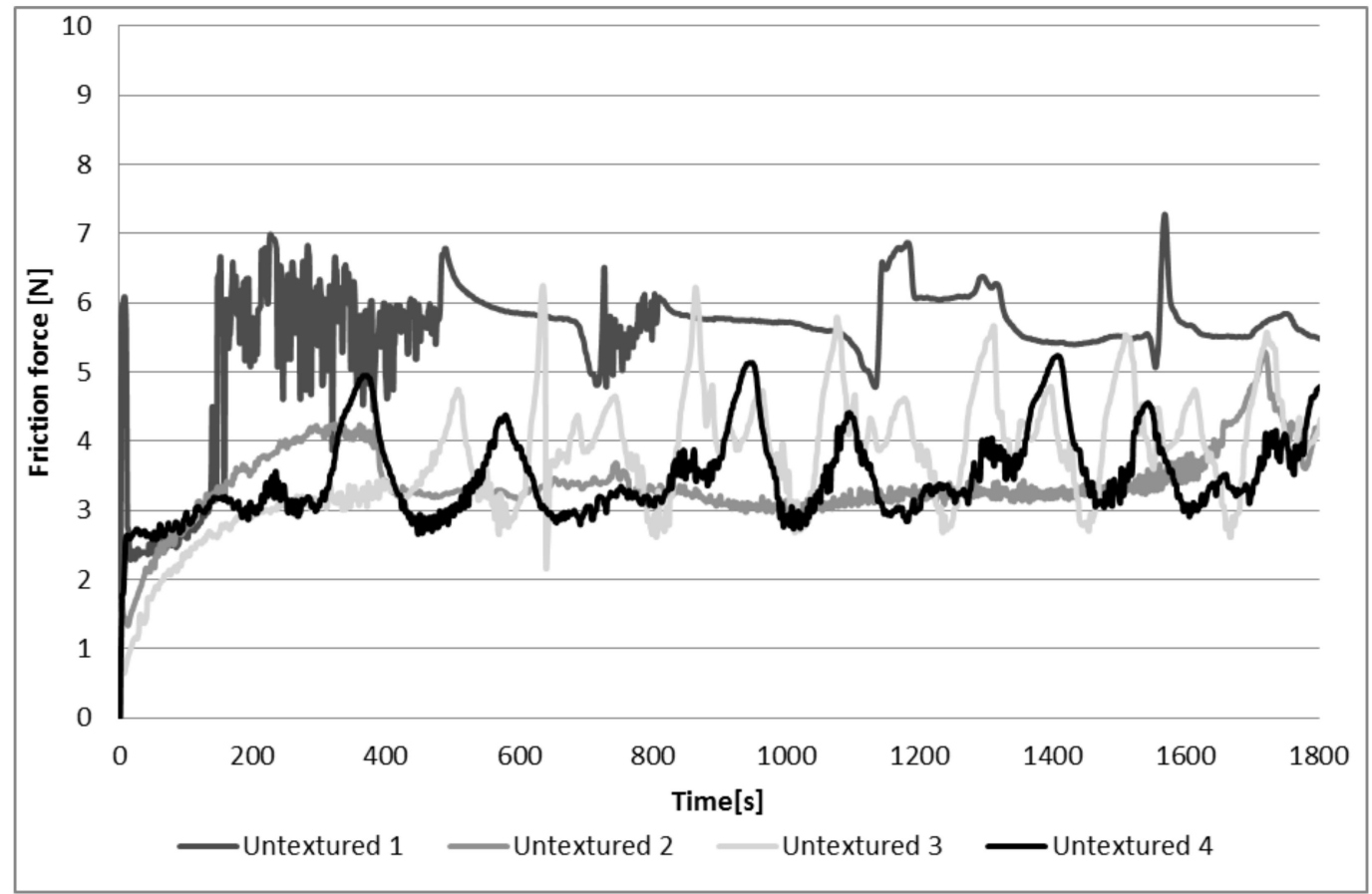

Figure 7. Friction force versus time for assembly with an untextured disc.

Figure 8 presents friction force runs for assemblies with textured disc of type 1.

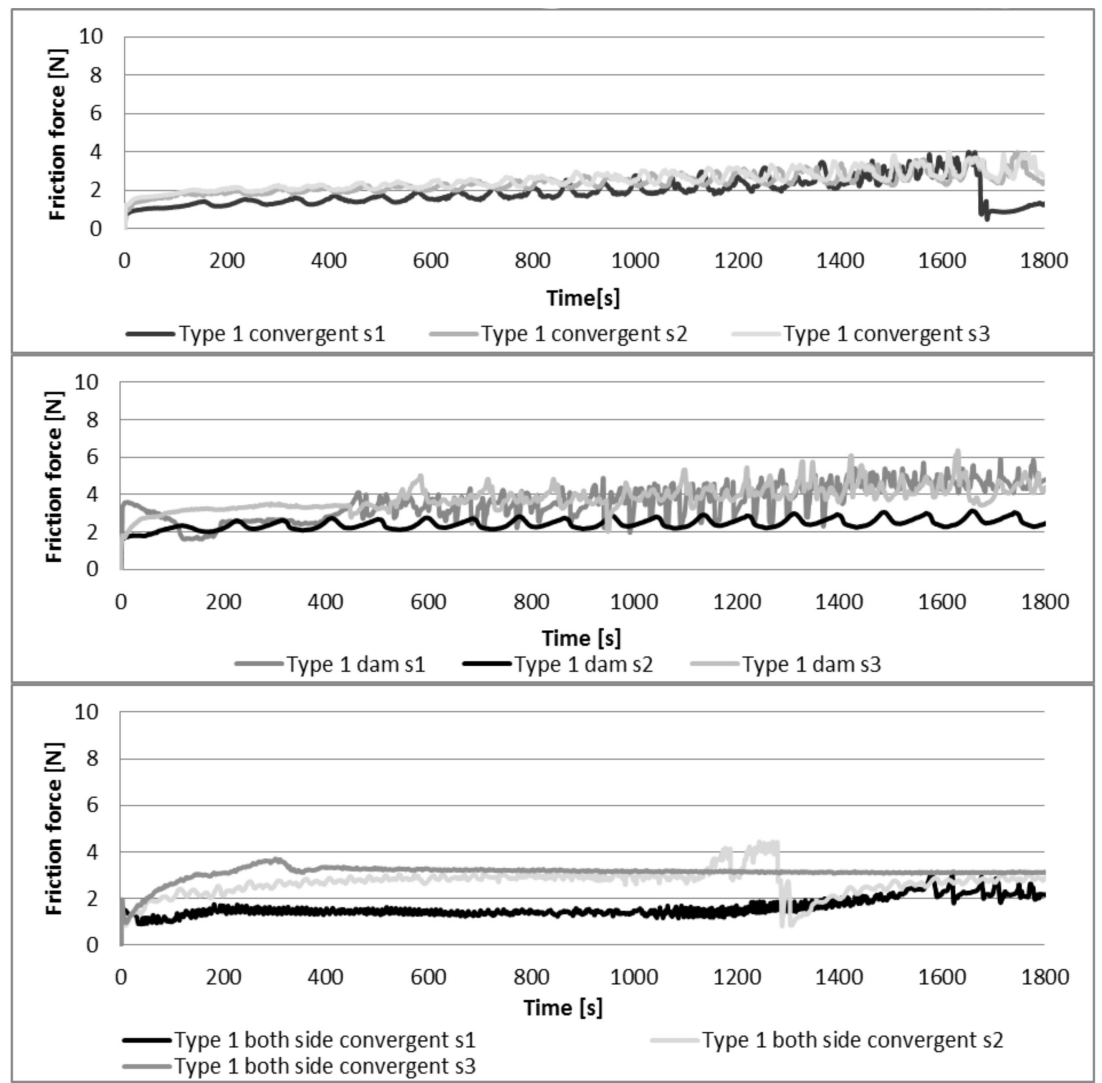

Figure 8. Cont. 


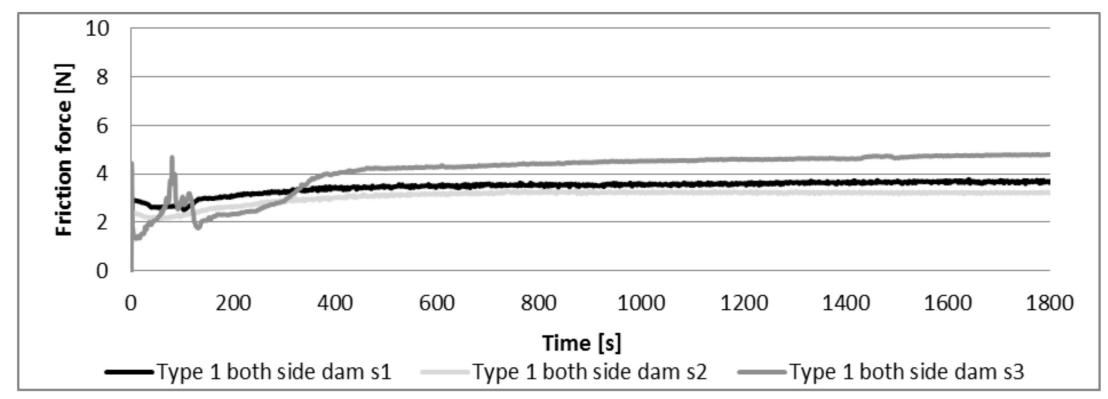

Figure 8. Friction force versus time for assemblies with a textured disc of type 1.

The course of the friction force curve for assembly with textured disc of type 1 with convergent bottom profile was different from that of sliding pair with untextured disc. The friction force initially sharply increased, obtained 1-1.5 $\mathrm{N}$ and then increased slowly. The final value of the friction force was $2.5-4 \mathrm{~N}$. The scatters of the friction force between test runs and within the same test run were smaller than those obtained for untextured assembly. The fluctuations of the friction force versus time (of the shape similar to sinusoid) were probably caused by a rotation of the counter-sample (small disc).

The change of the bottom profile from convergent to dam caused higher scatter of the friction force. In one test run the friction force increased abruptly, obtained the value about $2 \mathrm{~N}$ and then increased very slowly - its final value was between 2.5 and $3 \mathrm{~N}$. In two other runs, the friction forces after obtaining value of about $3 \mathrm{~N}$ increased, but more quickly than in the mentioned latter test run and finally obtained values between 4 and $6 \mathrm{~N}$. In both cases scatters of the friction forces within test run were comparatively large.

When both sides of the groove bottom profile were convergent, the final values of the friction forces were comparatively small: 2-3 N. However the shape of the friction force curves varied. In one test run the friction force was stable after about 300 seconds. In other cases the friction forces after initial sharp growths slowly increased. The fluctuations of the friction forces within one test run were lower than in the analysed previously cases.

A low variation of the friction force within the same test run was also observed for sliding pairs containing both sides dam of the profile bottom in the middle and final parts of tests. The friction forces after initial changes slowly increased and obtained the final values between 3.2 and $4 \mathrm{~N}$; they were higher compared to those obtained for the disc with convergent bottom shape from the both sides.

Figure 9 shows average friction coefficients with scatters for sliding pairs with untextured disc and discs with straight radial groves (of type 1) during the whole tests as well as at the beginning and at the end of tests. Values described as "the beginning of the test" were calculated as average values between 60 and 360 seconds of the test. Average values calculated between 1500 and $1800 \mathrm{~s}$ were described as "end of the test". Average values at "whole test" were calculated between 60 and 1800 seconds. The first 60 seconds of each test were discarded due to instabilities of the friction forces. One can see that the worst tribological performance was obtained for the assembly with untextured disc. In this case both the mean value and the scatter of the coefficient of friction were the largest. Textured discs led to lower frictional resistance particularly in the initial test parts. The convergent profile bottoms from one and both sides seem to be better solution than the profile bottom of dam shape. However, taking the scatter into consideration, there is difficult to find any difference between the behaviors of textured samples of type 1 .

Figure 10 presents curves characterizing the friction force versus time for assemblies with spiral discs of types 2 and 3. 


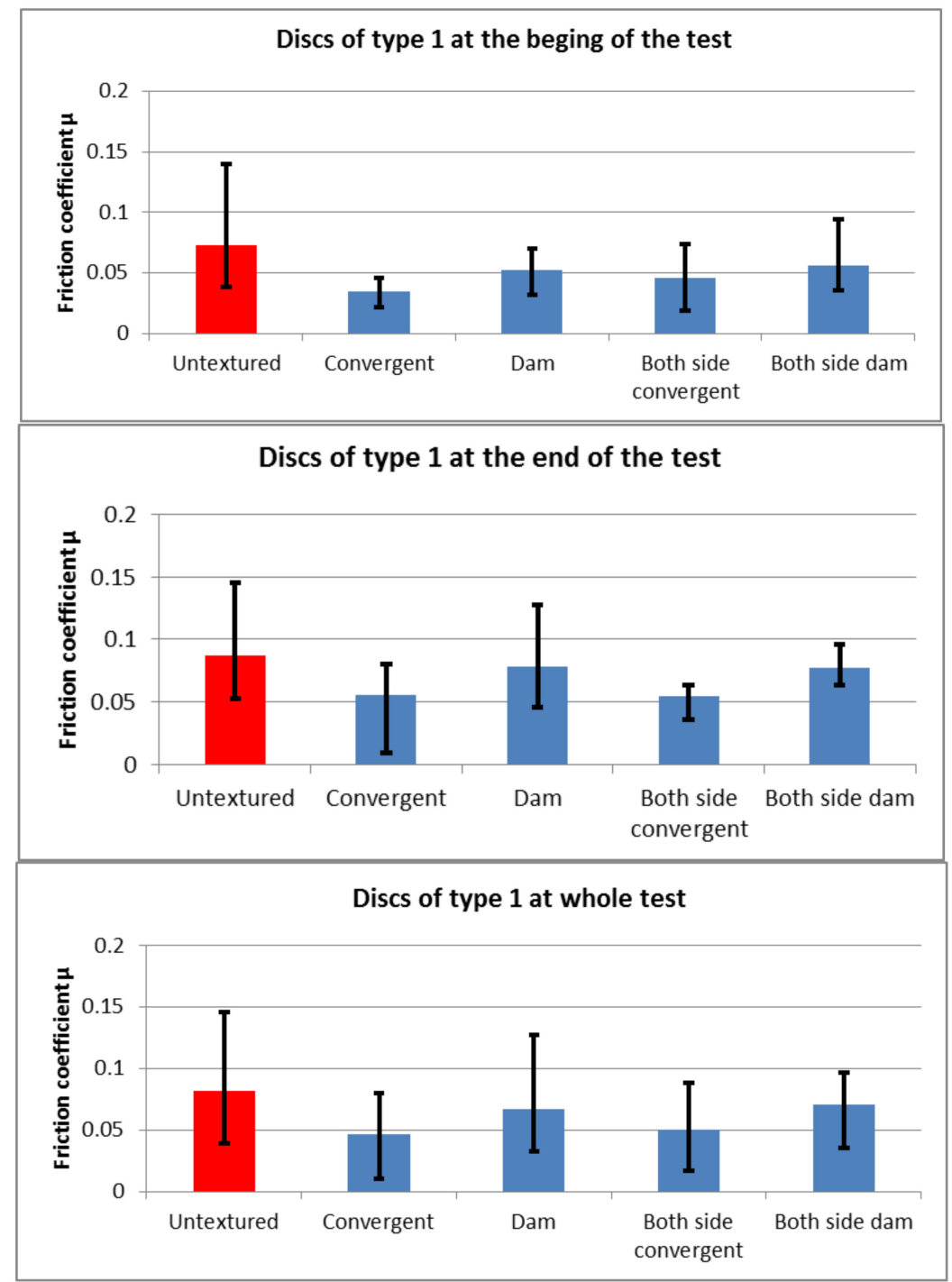

Figure 9. Average friction coefficients with scatters for assemblies with an untextured disc and discs with straight radial grooves (of type 1).

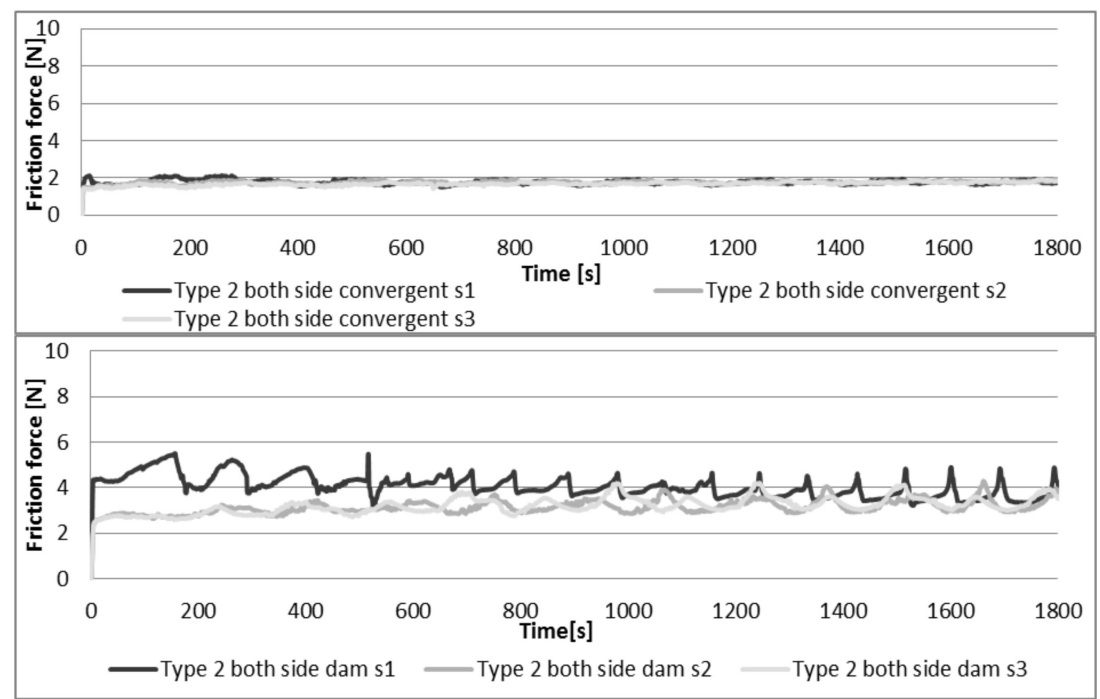

Figure 10. Cont. 


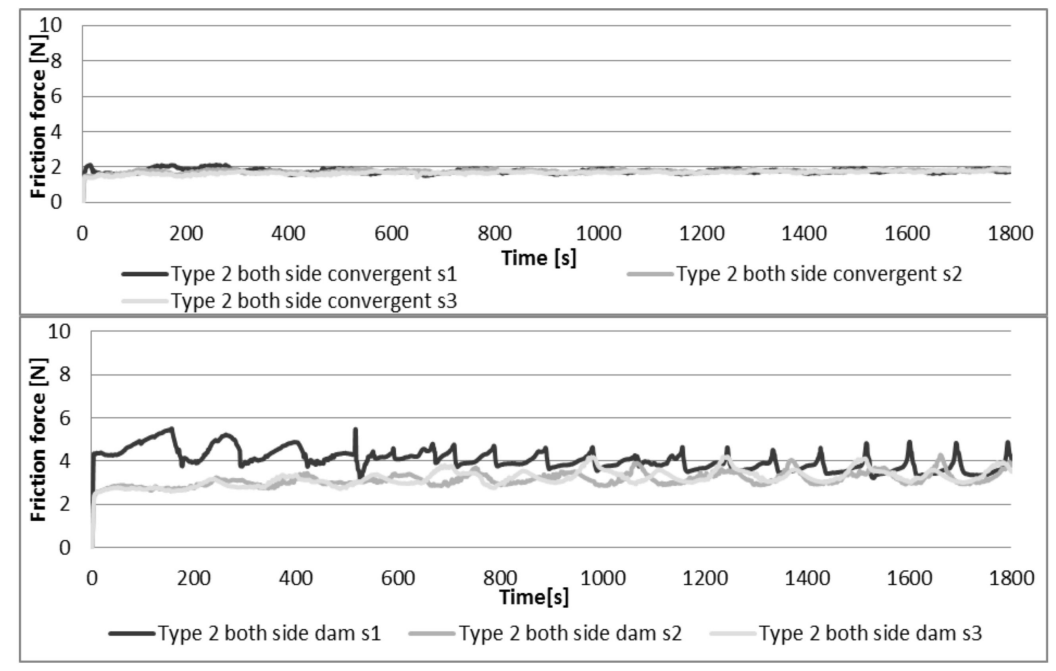

Figure 10. Friction force versus time for assemblies with a textured discs of type 2 and type 3 .

A convergent disc of type 2 after initial sharp increase in a few seconds obtained stable value a little smaller than $2 \mathrm{~N}$. The fluctuation of the friction force was low.

Changing the bottom profile from convergent to dam for the disc of type 2 caused an increase in the friction force, its variation also increased between and within test runs. After a few seconds the friction force obtained values of 3-4 $\mathrm{N}$ and then in two runs the friction force marginally increased, while in the third case - decreased. The final value of the friction force was between 3 and $5 \mathrm{~N}$.

Beneficial results were achieved for both side convergent disc of type 3 . In two cases the fiction force after obtaining a constant value was stable. Its final value was between 1.3 and $1.6 \mathrm{~N}$. The fluctuations of the friction forces were small.

Changes of the shape of the groove bottom from convergent to dam led to increases in both the friction force values and scatters. The final value of the friction force was between 2 and $4 \mathrm{~N}$.

In general the convergent bottom profile of the groove led to better results for discs of types 2 and 3 compared to dam bottom shape. The courses of the friction force curves in all analyzed cases were superior to those found for sliding pairs with untextured samples and with the disc of type 1.

Figure 11 shows graphs presenting the friction force versus time for assemblies with textured discs of types 4 and 5 .

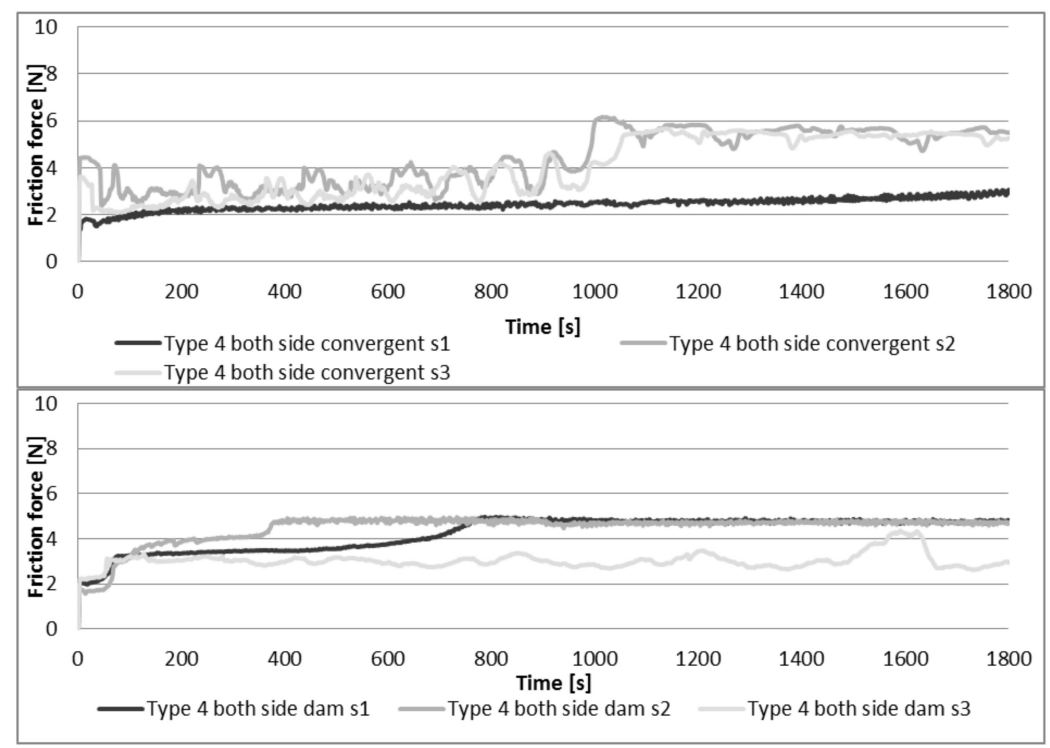

Figure 11. Cont. 


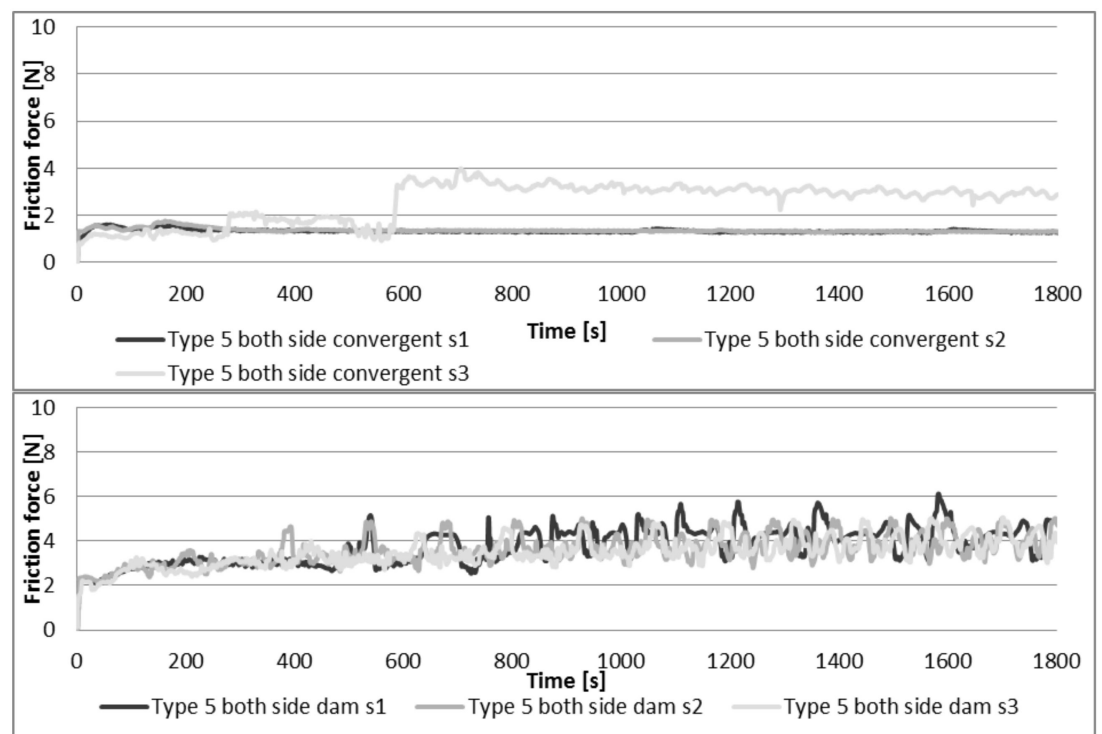

Figure 11. Friction force versus time for assemblies with textured discs of type 4 and type 5 .

The disc of type 4 gave not good tribological results. For both profiles of the bottom of the groove scatters of the friction force were comparatively high. When this profile was convergent, the friction force increased with time. The final value of the friction force was between 2.8 and $5.5 \mathrm{~N}$. When this bottom has dame shape after 800 seconds the friction force was constant, especially in two cases. The final friction force was between 2.7 and $4.8 \mathrm{~N}$. Generally, both profiles of the bottom of grooves gave similar results.

The friction force corresponding to the disc with convergent groove bottom profile of type 5 after initial sharp increase in a few seconds obtained stable value near $1 \mathrm{~N}$. In two cases the friction force increased to $1.3 \mathrm{~N}$ and was stable during the test; the fluctuation of the friction force was low. In the third run the friction force after about 300 seconds abruptly increased to $3.5 \mathrm{~N}$ and then slowly decreased. The final value of the friction force was between 1.3 and $3 \mathrm{~N}$.

Changing the groove bottom profile from convergent to dam for the disc of type 5 caused an increase in the friction force values and scatters. After a few seconds the friction force obtained the value about $2 \mathrm{~N}$ and then it slowly increased and obtained the final value between 3.5 and $5 \mathrm{~N}$. The convergent bottom profile of the groove was better for the disc of type 5 .

Figure 12 shows average friction coefficients with scatters for textured discs with spiral grooves of types 2, 3, 4, and 5 during the whole test as well as at the beginning and at the end of tests. Since, in most cases, the friction force was nearly constant during the test, three graphs were similar. The smaller scatter of the friction force was obtained in the final parts of tests. The best results (the smallest friction forces values and scatters) were achieved for the discs of type 2 and 3 with convergent groove profile bottoms. For the disc of type 5 the convergent profile bottom of the groove led to better results, however, the scatter of the friction force was comparatively high. The worst results (high friction forces values and scatters) were obtained for the disc of type 4 independently of its groove bottom profile.

During tests the truncation of the highest asperities occurred. However, wear was smaller than the height of the original surface roughness - Figure 13. The wWear level was comparatively high for untextured samples and textured samples with dam groove bottom shapes, particularly for the radial pattern of grooves. 

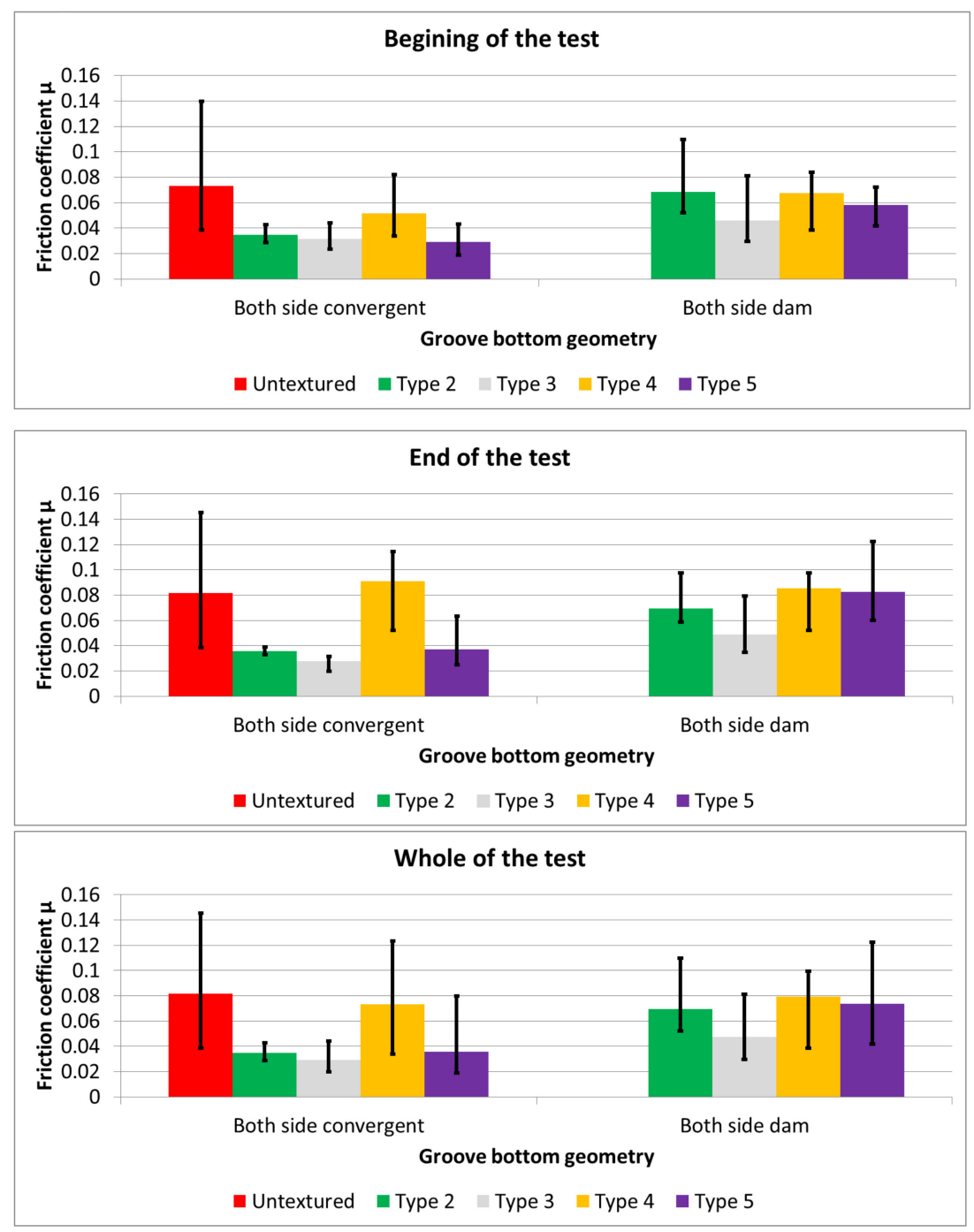

Figure 12. Average friction coefficients with scatters for assemblies with textured discs with spiral grooves (of types 2, 3, 4, and 5).

The untextured disc led to high values of the coefficient of friction values and, perhaps more importantly, the highest scatters both within the test run and between test runs. This performance was caused by disc surface topography after grinding. Because of the lack of oil pockets, the assembly with untextured disc operated in boundary lubrication conditions or in mixed lubrication conditions with dominance of boundary lubrication regions over full lubrication areas, in this case the formation of the hydrodynamic lift was not possible. An increase in the average temperature of tested assembly, due to friction force, was also the greatest for untextured discs $\left(8^{\circ} \mathrm{C}\right)$. For comparison, the average temperature increases of the assemblies with textured discs leading to the greatest and the lowest friction coefficients were 3.2 and $2.4{ }^{\circ} \mathrm{C}$, respectively. However, due to the high distance between the friction region and the temperature sensor most of heat generated was dispersed. To gain more precise data a thermal imaging camera should be used.

Situation was improved after introducing radial grooves (disc of type 1). A reduction in the frictional resistance due to surface texturing was the largest in the initial part of the test. An increase in the friction force as test progressed obtained for sliding pairs with textured samples was a problem. This behavior was probably caused by the oil loss. During tests straight radial grooves presumably gathered the lubricant, which leaked out from the contact zone due to a centrifugal force presence. However, in this case a reduction of the resistance to motion and the smaller friction force variation occurred, compared to the assembly with untextured disc sample. The grooves with convergent bottom 
profiles (one side and both sides) were slightly superior to those with the dam shape. The obtained values of the friction coefficient confirmed the known ability to decrease friction with the use of the convergent shape of the friction surfaces due to the formation of the lubrication wedge. During tests the amount of oil was sufficient to fill all the grooves; moreover, there was no visible wear debris entrapped inside the grooves after the test. Significantly longer tests could probably show a positive effect of the grooves as traps for wear debris.

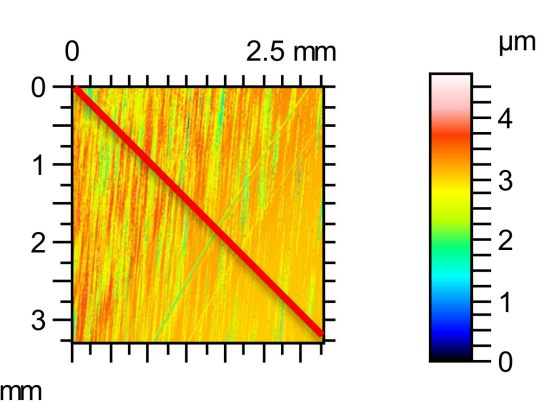

(a)

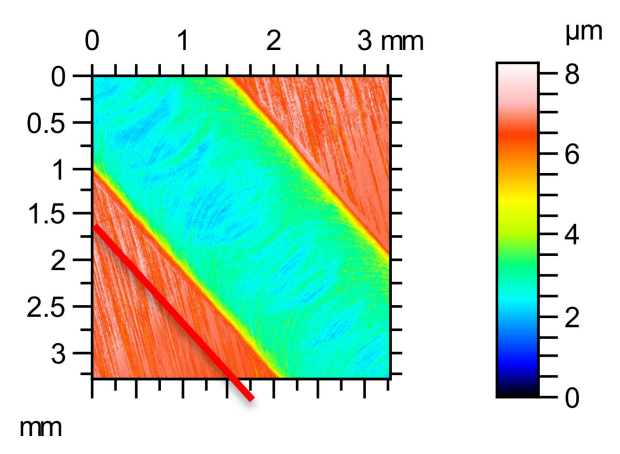

(c)

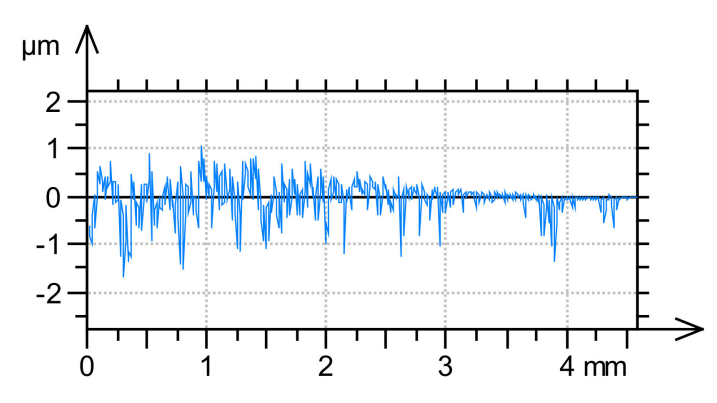

(b)

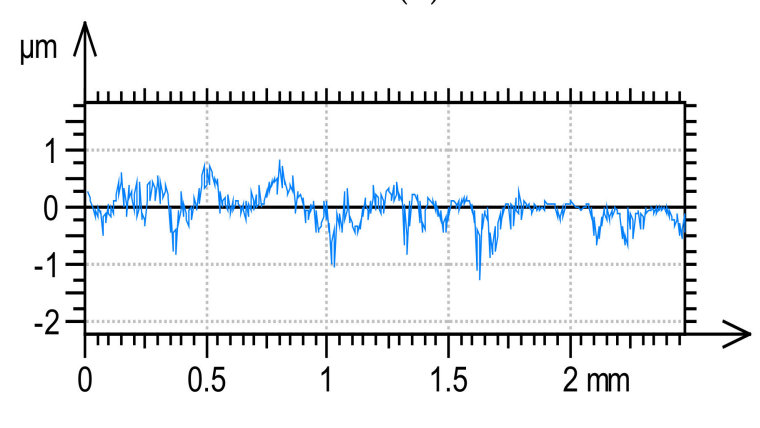

(d)

Figure 13. Contour plots $(\mathbf{a}, \mathbf{c})$ and extracted profiles $(\mathbf{b}, \mathbf{d})$ of worn untextured disc surface $(\mathbf{a}, \mathbf{b})$ and surface of type 1 with both side dam bottom shape of the groove $(\mathbf{c}, \mathbf{d})$.

Spiral discs of types 2,3,4, and 5 were created to gather the oil to the contact area and to reduce oil loss. Better tribological behaviors of spiral pattern of dimples than those of radial rows layout of oil pockets were also achieved in work [39]. In most cases the friction force did not increase during tests, especially for the convergent groove bottom profile. For discs of types 2,3, and 5 the convergent profile bottom of the groove was superior to that of the dam bottom shape. The tribological performance of the disc of type 4 was independent of the shape of the disc texture bottom. The smallest frictional force and the best stability of results were achieved for the disc of type 2. The disc of type 3 of both side convergent groove bottom profile assured also stable and small resistance to motion. The disc of type 5 of both side convergent groove bottom shape also led to comparatively small friction force, but the scatter of the coefficient of friction was high. The worst results were obtained when the disc of type 4 was used: high resistance to motion and high fluctuations of the friction force occurred.

To explain the results of experimental investigations during the contact of discs with spiral grooves with the counter-samples, the additional analysis was conducted (Figure 14).

The spiral fragment located in the contact zone depends on the curvature of the spiral used. The texture of type 5 generated the equal flows of the lubricant inside the groove to the inner and outer sides of the disc (Figure 14a). When discs 2 and 3 were used, the flow inside the groove generated by rotational movement would be directed into the center of the disc (Figure 14b), this solution helps to decrease oil loss from the contact zone due to flow generated by the centrifugal force. The texture of type 4 generated the flow outside the disc (Figure 14c). One can see from this analysis that the 
performance of discs of type 4 should be the worst, due to the largest oil loss. These assumptions were confirmed for assemblies containing textures with both side convergent bottoms of grooves. For grooves with both side dam bottoms the hydrodynamic force was not generated so easily and therefore the effect of spiral pattern vanished. Therefore, in most cases the convergent profile of groove bottom gave better results than dame bottom shape. These results are in accordance with other works; Galda et al. [30] and Lu et al. [31,32] achieved beneficial tribological effects of converging profiles of isolated dimples bottoms.

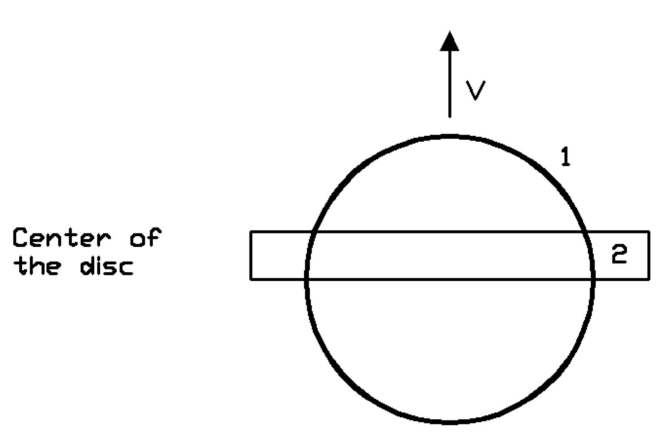

(a)

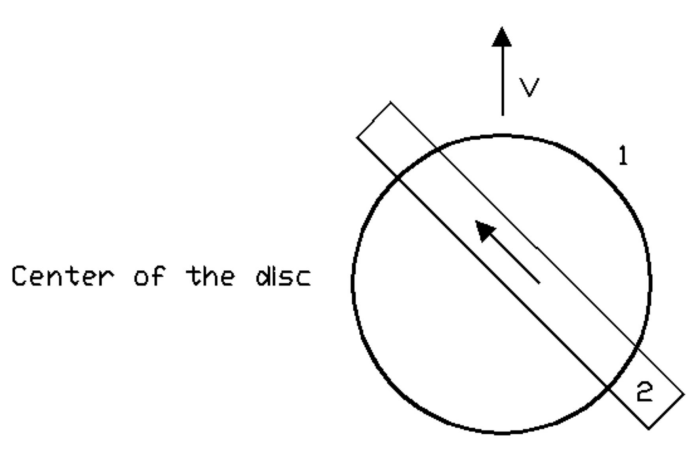

(b)

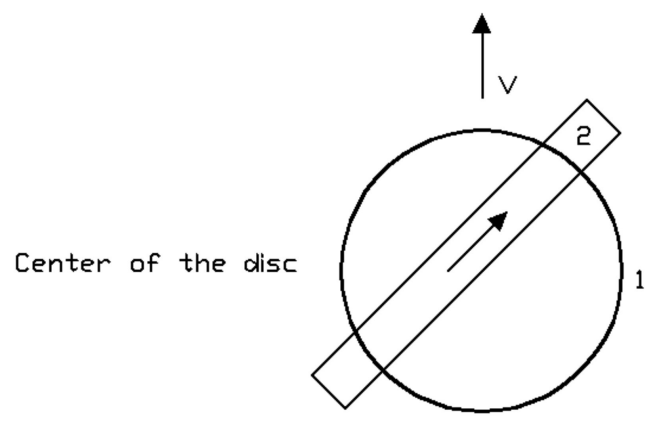

(c)

Figure 14. Scheme of oil flow inside the groove depending on the contact between the spiral fragment and the counter-sample; V- linear speed, 1-the contact area with the counter-sample, 2-the position of the spiral groove; (a) equal flows of the lubricant, (b) the flow directed to the center of the disc, and (c) flow outside the disc.

Although the shapes of spirals were created to gather oil in one direction of rotation, only the spiral fragments located in the friction zones affected oil gathering. Therefore, the triblogical performances of discs of types 4 and 5 were not so beneficial as presumed.

The effect of oil loss due to the presence of the centrifugal force on the coefficient of friction was found to be substantial in other research concerning surface texturing [28]. Grützmacher et al. [42] also noticed the increase in friction in lubricated sliding as a result of centrifugal force presence.

\section{Conclusions}

Surface texturing was found to be beneficial for the reduction of the friction coefficient up to $55 \%$ in comparison to untextured surface.

Convergent profile of the groove bottom was beneficial in most cases especially when is connected with the spiral pattern of the grooves. The best results were achieved for samples with both side convergent groove bottom and the disc patterns of types 2 and 3 . 
Simple shape of the groove (type 1) in most cases led to high scatter of the friction coefficient. This phenomenon was probably caused by constant losses of lubricant due to the presence of the centrifugal force. Spiral grooves helped to gather oil into the contact area, which led to decreases in the friction coefficient value and scatter at the end of test.

Author Contributions: Conceptualization: S.W., W.K., A.D., P.P.; methodology: S.W., W.K., A.D., P.P.; investigation, and formal analysis: S.W., W.K., A.D., P.P.; writing-original draft preparation: S.W., W.K., A.D., P.P.; writing-review and editing: S.W., W.K., A.D., P.P. All authors have read and agreed to the published version of the manuscript.

Funding: This research was partially funded by the National Science Centre (decision no. 2018/31/B/ST8/02946).

Conflicts of Interest: The authors declare no conflict of interest.

\section{References}

1. Etsion, I. State of the art in laser surface texturing. J. Tribol. Trans. ASME 2005, 125, 248-253. [CrossRef]

2. Nilsson, B.-G.; Rosen, T.R.; Thomas, D.; Wiklund. Oil pockets and surface topography: Mechanism of friction reduction. In Proceedings of the XI International Colloquium on Surfaces, Chemnitz, Germany, 2-3 February 2004.

3. Erdemir, A. Review of engineered tribological interfaces for improved boundary lubrication. Tribol. Int. 2005, 38, 249-256. [CrossRef]

4. Gachot, C.; Rosenkranz, A.; Hsu, S.M.; Costa, H.L. A critical assessment of surface texturing for friction and wear improvement. Wear 2017, 372-373, 21-41. [CrossRef]

5. Rosenkranz, A.; Grützmacher, P.G.; Gachot, C.; Costa, H.L. Surface texturing in machine elements-a critical discussion for rolling and sliding contacts. Adv. Eng. Mater. 2019, 21, 1900194. [CrossRef]

6. Anno, J.N.; Walowit, J.A.; Allen, C.M. Microasperity lubrication. J. Tribol. 1968, 90, 351-355. [CrossRef]

7. Li, T.; Lu, X.; Ma, X.; Xu, H.; Jiao, B.; Zou, D. Numerical and experimental analysis of the honing texture on the lubrication performance of piston ring-cylinder liner tribosystem. Tribol. Trans. 2019, 62, 991-1006. [CrossRef]

8. Anderberg, C.; Dimkovski, Z.; Rosén, B.-G.; Thomas, T.R. Low friction and emission cylinder liner surfaces and the influence of surface topography and scale. Tribol. Int. 2019, 133, 224-229. [CrossRef]

9. Mezghani, S.; Demirci, I.; Zahouani, H.; El Mansori, M. The effect of groove texture patterns on piston-ring pack friction. Precis. Eng. 2012, 36, 210-217. [CrossRef]

10. Grabon, W.; Pawlus, P.; Wos, S.; Koszela, W.; Wieczorowski, M. Effects of honed cylinder liner surface texture on tribological properties of piston ring-liner assembly in short time tests. Tribol. Int. 2017, 113, 137-148. [CrossRef]

11. Zeng, S.; Li, J.; Zhou, N.; Zhang, J.; Yu, A.; He, H. Improving the wear resistance of PTFE-based friction material used in ultrasonic motors by laser surface texturing. Tribol. Int. 2020, 141, 105910. [CrossRef]

12. Wang, M.; Zhang, C.; Wang, X. The wear behavior of textured steel sliding against polymers. Materials 2017, 10, 330. [CrossRef] [PubMed]

13. Kasem, H.; Stav, O.; Grützmacher, P.; Gachot, C. Effect of low depth surface texturing on friction reduction in lubricated sliding contact. Lubricants 2018, 6, 62. [CrossRef]

14. Braun, D.; Greiner, C.; Schnider, J.; Gumbsch, P. Efficiency of laser surface texturing in the reduction of friction under mixed lubrication. Tribol. Int. 2014, 77, 142-147. [CrossRef]

15. Xiong, D.; Qin, Y.; Li, J.; Wan, Y.; Tyagi, R. Tribological properties of PTFE/laser surface textured stainless steel under starved oil lubrication. Tribol. Int. 2015, 82, 305-310. [CrossRef]

16. Pettersson, U.; Jacobson, S. Friction and wear properties of micro textured DLC coated surfaces in boundary lubricated sliding. Tribol. Lett. 2004, 17, 553-559. [CrossRef]

17. Moronuki, N.; Furukawa, Y. Frictional properties of the micro-textured surface of anisotropically etched silicon. CIRP Ann. Manuf. Technol. 2003, 52, 471-474. [CrossRef]

18. Yuan, S.; Huang, W.; Wang, X. Orientation effects of micro-grooves on sliding surfaces. Tribol. Int. 2011, 44, 1047-1054. [CrossRef]

19. Ren, N.; Nanbu, T.; Yasuda, Y.; Zhu, D.; Wang, Q. Micro textures in concentrated-conformal-contact lubrication: Effect of distribution patterns. Tribol. Lett. 2007, 28, 275-285. [CrossRef] 
20. Zum Gahr, K.H.; Wahl, R.; Wauthier, K. Experimental study of the effect of microtexturing on oil lubricated ceramic/steel friction pairs. Wear 2009, 267, 1241-1251. [CrossRef]

21. Costa, H.L.; Hutchings, M. Effects of die surface patterning on lubrication in strip drawing. J. Mater. Process. Technol. 2009, 209, 1175-1180. [CrossRef]

22. Duarte, M.; Lasagni, A.; Giovanelli, R.; Narciso, J.; Louis, E.; Mücklich, F. Increasing lubricant film lifetime by grooving periodical patterns using laser interference metallurgy. Adv. Eng. Mater. 2008, 10, 554-558. [CrossRef]

23. Michail, S.K.; Barber, G.C. The effects of roughness on piston ring lubrication-Part II: The relationship between cylinder wall surface topography and oil film thickness. Tribol. Trans. 1995, 38, 173-177. [CrossRef]

24. Bolander, N.W.; Sadeghi, H. Deterministic modeling of honed cylinder liner friction. Tribol. Trans. 2007, 50, 248-256. [CrossRef]

25. Costa, H.L.; Hutchings, I.M. Hydrodynamic lubrication of textured steel surfaces under reciprocating sliding conditions. Tribol. Int. 2007, 40, 1227-1238. [CrossRef]

26. Vladescu, S.-C.; Olver, A.V.; Pegg, G.; Reddyhoff, T. The effects of surface texture in reciprocating contacts-an experimental study. Tribol. Int. 2015, 82, 28-42. [CrossRef]

27. Shen, C.; Khonsari, M.M. Texture shape optimization for seal-like parallel surfaces: Theory and experiment. Tribol. Trans. 2016, 59, 698-706. [CrossRef]

28. Wos, S.; Koszela, W.; Pawlus, P. Comparing tribological effects of various chevron-based surface textures under lubricated unidirectional sliding. Tribol. Int 2020. In Press. [CrossRef]

29. Wang, W.; Huang, Z.; Shen, D.; Kong, L.; Li, S. The effect of triangle-shaped surface textures on the performance of the lubricated point-contacts. J. Tribol. 2013, 135, 1-11. [CrossRef]

30. Galda, L.; Dzierwa, A.; Sep, J.; Pawlus, P. The effect of oil pockets shape and distribution on seizure resistance in lubricated sliding. Tribol. Lett. 2010, 37, 301-311. [CrossRef]

31. Lu, P.; Wood, R.J.K.; Gee, M.; Wang, L.; Pfleging, W. A novel surface texture shape for directional friction control. Tribol Lett. 2018, 66, 51. [CrossRef]

32. Lu, P.; Wood, R.J.K.; Gee, M.; Wang, L.; Pfleging, W. The use of anisotropic texturing for control of directional friction. Tribol. Int. 2017, 113, 169-181. [CrossRef]

33. Costa, H.L.; Hutchings, I.M. Some innovative surface texturing techniques for tribological purposed. Proc. Inst. of Mech. Eng. Part J 2015, 229, 429-444. [CrossRef]

34. Grützmacher, P.I.; Profito, F.J.; Rosenkranz, A. Multi-scale surface texturing in tribology—Current knowledge and future perspectives. Lubricants 2019, 7, 95. [CrossRef]

35. Ancona, A.; Joshi, G.; Volpe, A.; Scaraggi, M.; Lugarà, P.; Carbone, G. Non-uniform laser surface texturing of an un-tapered square pad for tribological applications. Lubricants 2017, 5, 41. [CrossRef]

36. Ancona, A.; Carbone, G.; De Filippis, M.; Volpe, A.; Lugarà, P.M. Femtosecond laser full and partial texturing of steel surfaces to reduce friction in lubricated contact. Adv. Opt. Technol. 2014, 3, 539-547. [CrossRef]

37. Nakano, M.; Korenaga, A.; Miyake, K.; Murakami, T.; Ando, Y.; Usami, H.; Sasaki, S. Applying micro-texture to cast iron surfaces to reduce the friction coefficient under lubricated conditions. Tribol. Lett. 2007, 28, 131-138. [CrossRef]

38. Wang, X.; Liu, W.; Zhou, F.; Zhu, D. Preliminary investigation of the effect of dimple size on friction in line contacts. Tribol. Int. 2009, 42, 1118-1123. [CrossRef]

39. Wos, S.; Koszela, W.; Pawlus, P. Determination of oil demand for textured surfaces under conformal contact conditions. Tribol. Int. 2016, 93, 602-613. [CrossRef]

40. Koszela, W.; Dzierwa, A.; Galda, L.; Pawlus, P. Experimental investigation of oil pockets effect on abrasive wear resistance. Tribol. Int. 2012, 46, 145-153. [CrossRef]

41. Tian, H.; Saka, N.; Suh, N.P. Boundary lubrication studies on undulated titanium surfaces. Tribol. Trans. 1989, 32, 289-296. [CrossRef]

42. Grützmacher, P.G.; Rosenkranz, A.; Rammacher, S.; Gachot, C.; Mücklich, F. The influence of centrifugal forces on friction and wear in rotational sliding. Tribol. Int. 2017, 116, 256-263. [CrossRef]

(C) 2020 by the authors. Licensee MDPI, Basel, Switzerland. This article is an open access article distributed under the terms and conditions of the Creative Commons Attribution (CC BY) license (http://creativecommons.org/licenses/by/4.0/). 